\title{
Post-weaning diet affects genomic imprinting at the insulin-like growth factor 2 (Igf2) locus
}

\author{
Robert A. Waterland ${ }^{1, *}$, Juan-Ru Lin ${ }^{1}$, Charlotte A. Smith ${ }^{1}$ and Randy L. Jirtle ${ }^{2}$ \\ ${ }^{1}$ Departments of Pediatrics and Molecular and Human Genetics, Baylor College of Medicine, USDA Children's \\ Nutrition Research Center, Houston, TX 77030-2600, USA and ${ }^{2}$ Department of Radiation Oncology, Duke University \\ Medical Center, Durham, NC 27710, USA
}

Received December 13, 2005; Revised and Accepted January 12, 2006 DDBJ/EMBL/GenBank accession nos AY849916-AY849923.

\begin{abstract}
IGF2 loss of imprinting (LOI) is fairly prevalent and implicated in the pathogenesis of human cancer and developmental disease; however, the causes of this phenomenon are largely unknown. We determined whether the post-weaning diet of mice affects allelic expression and CpG methylation of Igf2. C57BL/ 6J $\times$ Cast/EiJ F1 hybrid mice were weaned onto (1) a standard natural ingredient control diet, (2) a synthetic control diet or (3) a synthetic methyl-donor-deficient diet lacking folic acid, vitamin $\mathrm{B}_{12}$, methionine and choline. Maternal Igf2 expression in kidney was negligible at birth, but increased to $\sim 10 \%$ of total expression after 60 days on the natural control diet. By 60 days post-weaning, both synthetic diets caused significant LOI of Igf2 relative to animals weaned onto the natural control diet. Total Igf2 expression was significantly reduced in these groups, however, indicating that the increase in relative maternal Igf2 expression was caused by specific down-regulation of the paternal allele. The LOI induced by the synthetic-deficient diet persisted during a subsequent 100-day 'recuperation' period on natural ingredient diet. There were no group differences in overall or allele-specific CpG methylation in the $\mathrm{H19}$ differentially methylated region (DMR), Igf2 DMR0 or Igf2 DMR1. At 30 and 60 days post-weaning, however, the paternal allele of Igf2 DMR2 was hypermethylated in the kidneys of mice on the control synthetic diet. These results indicate that postweaning diet can permanently affect expression of Igf2, suggesting that childhood diet could contribute to IGF2 LOI in humans.
\end{abstract}

\section{INTRODUCTION}

Epigenetic mechanisms maintain mitotically and/or meiotically heritable differences in gene expression that are not dependent on DNA sequence alterations. For example, the diverse patterns of gene expression in the different tissues of complex organisms are established and maintained via programmed alterations in $\mathrm{CpG}$ methylation and various histone modifications that concertedly regulate the chromatin conformation and transcriptional activity of specific genomic regions (1). Just as genetic variation among individuals causes individual differences in gene expression and susceptibility to various diseases, it is becoming increasingly clear that interindividual differences in epigenetic gene regulation also influence susceptibility to disease $(2-4)$.

Although relatively few in number, genomically imprinted genes appear to play a disproportionately important role in human epigenetic disease $(3,5)$. Genomic imprinting is an epigenetic phenomenon whereby specific mammalian genes are expressed preferentially from the allele inherited either from the father or from the mother. The maternally inherited allele of the imprinted gene encoding insulin-like growth factor $2(I G F 2)$ is normally epigenetically silenced, resulting in expression almost exclusively from the paternal allele. Loss of imprinting (LOI) at the $I G F 2$ locus results in biallelic expression of this mitogenic growth factor in approximately $10 \%$ of normal human adults $(6,7)$ and is implicated in several types of cancer $(5,8)$ and Beckwith-Wiedemann syndrome (9). In most individuals, the cause of IGF2 LOI is unknown (10).

$\operatorname{Ig} f 2$ allelic expression in mice is regulated by allele-specific methylation at four differentially methylated regions (DMRs) (11). The H19 DMR upstream of the H19 gene (which is $90 \mathrm{~kb}$ $3^{\prime}$ of $\operatorname{Ig} f 2$ ) acts as a methylation-sensitive boundary element

*To whom correspondence should be addressed at: Baylor College of Medicine, USDA Children's Nutrition Research Center, 1100 Bates Street, Ste 5080, Houston, TX 77030-2600, USA. Tel: +1 7137980304; Fax: +1 7137987101; Email: waterland@bcm.edu

(C) The Author 2006. Published by Oxford University Press. All rights reserved.

For Permissions, please email: journals.permissions@oxfordjournals.org 


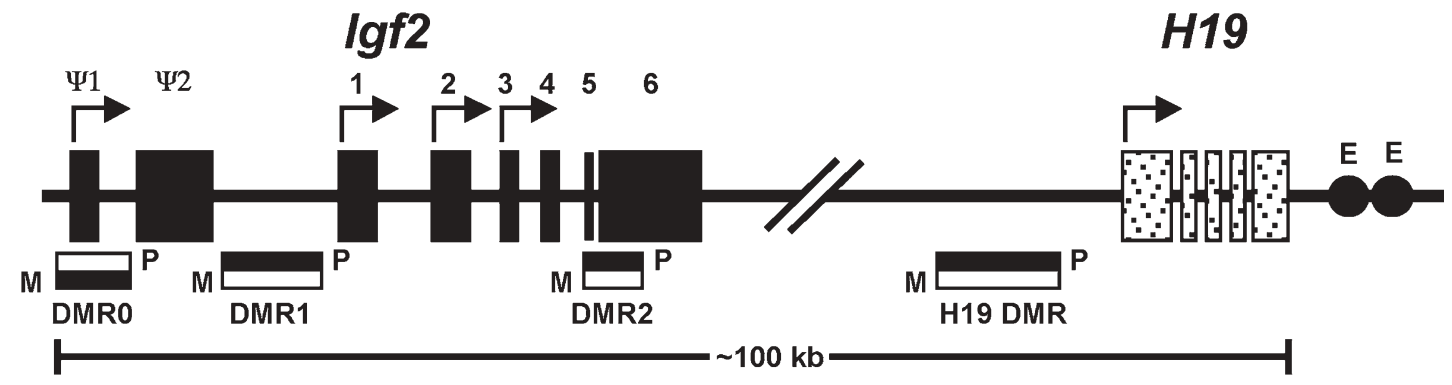

Figure 1. The mouse Igf2/H19 imprinted locus, indicating the location of four DMRs known to contribute to Igf2 imprinting. Boxes denoting the DMRs are shaded to indicate preferential methylation of the maternal (M) or paternal (P) allele in each region [adapted from Lopes et al. (11)].

and is normally methylated only on the paternal allele (Fig. 1). DMR0 overlaps a placenta-specific promoter of $\operatorname{Ig} 2$ and is methylated only on the maternal allele. Both DMR1, a methylation-sensitive silencer element upstream of the primary $I g f 2$ promoters, and DMR2, a methylation-sensitive activator within the last exon of $I g f 2$, are preferentially methylated on the paternal allele. Importantly, human genomic regions corresponding to the mouse H19 DMR (12), Igf2 DMR0 (13) and Igf2 DMR2 (14) have been identified and exhibit allele-specific methylation. Alterations in the methylation status of the genomic regions corresponding to the murine H19 DMR and Igf2 DMR0 have been implicated in IGF2 LOI in humans $(15,16)$. Such associations have yet to be reported for the human genomic region corresponding to mouse Igf2 DMR2.

Because of the unique epigenetic requirements associated with allele-specific expression, genomically imprinted genes may be especially sensitive to epigenetic dysregulation by environmental influences during development (17-19). Indeed, recent results from animal models and human epidemiologic studies support this conjecture. For example, subtle differences in the medium used to culture pre-implantation mouse embryos (20) and genetically engineered elevations in the activity of the maintenance methylase DNA methyltransferase 1 in mouse embryonic stem cells (21) affect methylation and expression at imprinted loci. Moreover, sporadic imprinting defects lead to Beckwith-Wiedemann syndrome and Angelman syndrome at significantly higher frequencies in children born following assisted reproductive technologies than in individuals conceived naturally (22). Taken together, these data indicate that the environment of the early embryo can affect the establishment and/or maintenance of epigenetic mechanisms regulating genomically imprinted genes.

Relatively little attention has been paid, however, to the possibility that the environmental lability of epigenetic regulation at genomically imprinted genes might continue during fetal development and even extend into the postnatal period. This possibility is of critical relevance to the 'early origins' hypothesis that environmental influences during development have a persistent effect on metabolism and health in adulthood (23). The rapid cellular proliferation during prenatal and early postnatal development requires a steady supply of methyl groups to maintain established patterns of allele-specific DNA methylation. Mammalian one-carbon metabolism relies upon dietary methyl donors and cofactors (including folic acid, vitamin $\mathrm{B}_{12}$, methionine and choline) to supply the methyl groups required for biological methylation reactions (24). Methyl donor nutrition during development may, therefore, wield a lasting influence on epigenetic gene regulation, a hypothesis recently validated in the viable yellow agouti mouse model (25).

In this study, we tested the hypothesis that dietary deficiency of methyl donors and cofactors during postweaning development will cause hypomethylation at Igf2 DMRs and consequent dysregulation of $I g f 2$ allelic expression. By comparing Igf2 allelic expression among animals weaned onto either a synthetic methyl-donor-deficient diet, a methyl-donor replete synthetic diet, or a natural ingredient (chow) diet, we found that dietary methyl donor deficiency during the post-weaning period caused dramatic LOI at the Igf 2 locus that persisted following a return to normal diet. We also report here three unexpected findings. First, Igf2 LOI, which was most prominent after 60 days on the synthetic diets, was actually associated with a decrease in total Igf2 expression, indicating specific down-regulation of the paternal allele rather than up-regulation of the maternal allele. Second, the Igf 2 LOI induced by dietary methyl donor deficiency occurred without detectable changes in methylation at any of the four DMRs known to regulate Igf2 allelic expression. Third, the methyl-donor replete synthetic diet induced $\operatorname{Igf} 2$ LOI comparable to that attributed to methyl donor deficiency and caused paternal allele-specific hypermethylation at Igf2 DMR2. These results demonstrate that nutritional stimuli in pre-adulthood can induce persistent epigenetic dysregulation of genomically imprinted genes. Thus, interindividual variation in human epigenotype and susceptibility to epigenetically based disease may be modified by nutrition during infancy and childhood.

\section{RESULTS}

\section{Methyl-deficient diet reversibly inhibits post-weaning weight gain}

$\mathrm{C} 57 \mathrm{BL} / 6 \mathrm{~J} \times \mathrm{Cast} / \mathrm{EiJ} \mathrm{F} 1$ offspring (hereafter referred to as C57BL/Cast F1) were weaned onto a natural ingredient control diet (NC), an amino-acid defined synthetic control diet (SC) or an amino-acid defined synthetic diet deficient in dietary methyl donors and cofactors (SD). Mice weaned onto the SC diet gained slightly less weight than those on the $\mathrm{NC}$ diet, whereas those weaned onto the SD diet gained 


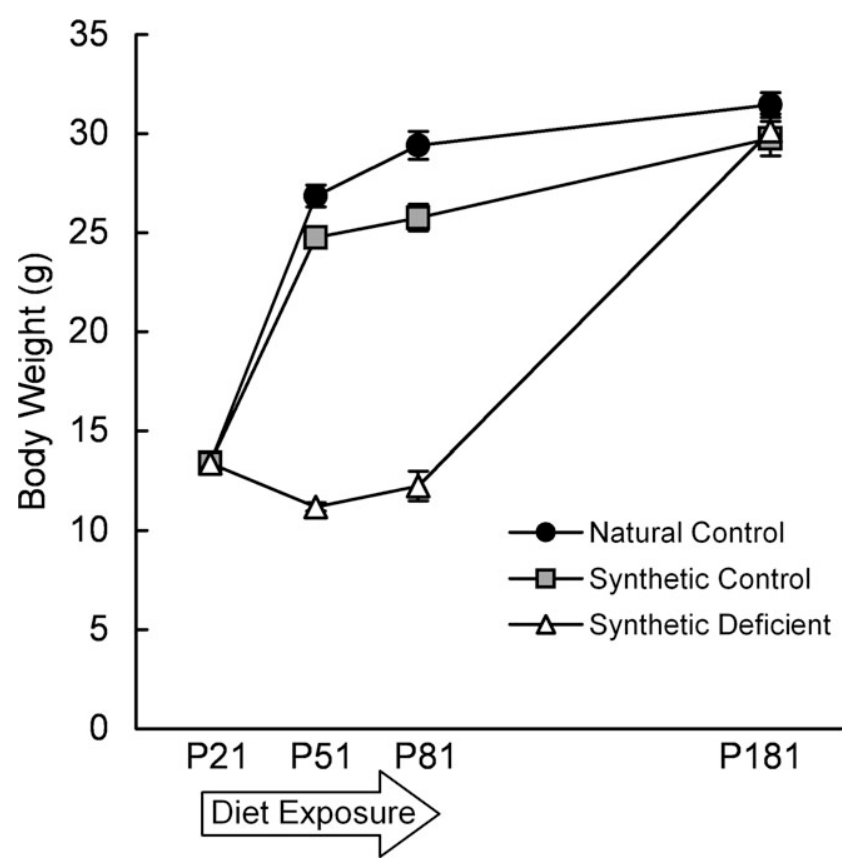

Figure 2. Body weight of male mice fed different diets for 60-day postweaning (mean \pm SEM of 2-4 animals per group at each age). Compared with mice fed the NC diet, mice fed the SC diet gained slightly less weight during the 60-day diet exposure from P21 to P81 $(P<0.05)$. Mice did not gain any weight on the SD diet $(P<0.001$ relative to $\mathrm{NC}$ at 30 and 60 days post-weaning). After the 100-day recuperation period (P81-P181), during which all animals received the NC diet, body weight did not differ significantly among the groups. Female mice showed similar group differences.

no weight during the 60-day diet exposure (Fig. 2). Following the 60-day differential diet exposure, mice from all three groups were provided the $\mathrm{NC}$ diet for an additional 100 days. During this 'recuperation' period, the average body weight of the SD group recovered completely to that of animals fed the $\mathrm{NC}$ diet from weaning (Fig. 2). Despite the dramatic effect on weight gain, mice fed the SD diet remained active and appeared healthy. There was no spontaneous mortality during the experiment.

\section{Developmental Igf2 LOI in kidney is affected by weanling diet}

To enable reliable quantitation of small but potentially biologically significant changes in allelic expression, we developed and validated an approach based on manual sequencing and phosphor-imager quantitation of Igf2 cDNA (Fig. 3). We examined overall $\operatorname{Ig} f 2$ expression in liver, kidney and lung of adult C57BL/Cast F1 mice. Igf2 expression in adult liver was nearly undetectable. Exploiting a C57BL/Cast SNP within $I g f 2$ exon 6 (26), we measured $\operatorname{Ig} f 2$ allelic expression in lung and kidney from animals at 60 days post-weaning and found significant group differences in kidney. Given the potential relevance of this model to IGF2 LOI in Wilms' tumor (15), subsequent studies were focused on kidney.

In kidney of C57BL/Cast F1 hybrids at embryonic day 18, maternal expression of $I g f 2$ was almost negligible (Fig. 4A). There was, however, substantial developmental loss of $\operatorname{Ig} 2$
A

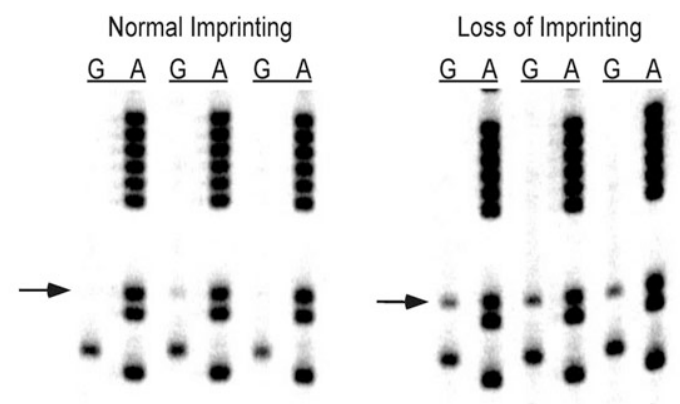

B

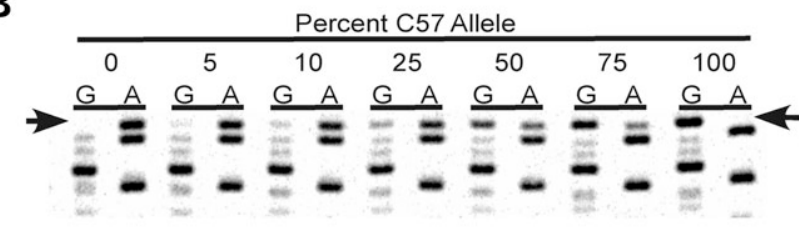

C

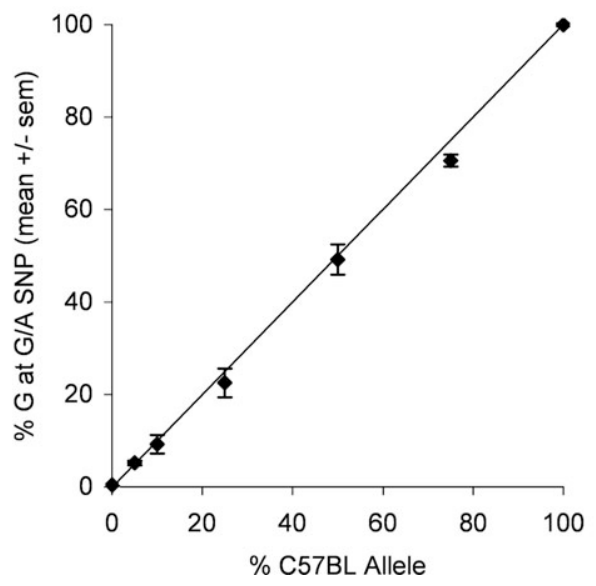

Figure 3. Direct sequencing method for measurement of $I g f 2$ allelic expression. (A) Gel images showing results of allelic sequencing of Igf2 cDNA in three animals showing maintenance of imprinting and three showing Igf2 LOI. The percentage of total Igf 2 expression derived from the maternal allele can be directly quantitated at the polymorphic site (arrow) as $100[\mathrm{G} /(\mathrm{A}+\mathrm{G})]$. (B) Gel image illustrating results of experiments sequencing through the Igf2 polymorphism in known mixtures of the C57BL and Cast alleles. The arrows indicate the polymorphic site. (C) Results of four independent mixing and sequencing experiments; the relationship between $\% \mathrm{G}$ at the G/A SNP and the \% C57BL allele in the C57BL:Cast mixture does not differ significantly from the line of identity (shown). Allelic expression as low as 5\% of total is clearly detectable.

imprinting in the postnatal period. In animals fed the $\mathrm{NC}$ diet throughout, relative expression of $I g f 2$ from the maternal allele increased to $10 \%$ of total by 30 days post-weaning and did not increase thereafter (Fig. 4A). Developmental loss of Igf2 imprinting was also influenced by weanling diet. Quite unexpectedly, the SC diet significantly increased $\operatorname{Ig} 22 \mathrm{LOI}$ at 30 and 60 days post-weaning (Fig. 4A). This effect did not persist through the 100-day recuperation period (Fig. 4A). By 60 days post-weaning, the SD diet also caused a significant 
A

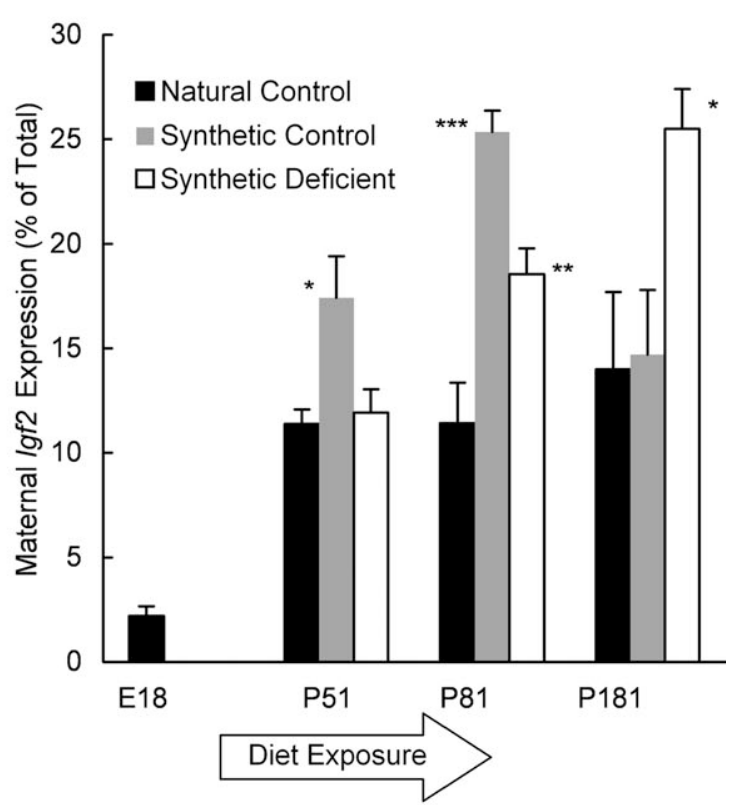

B

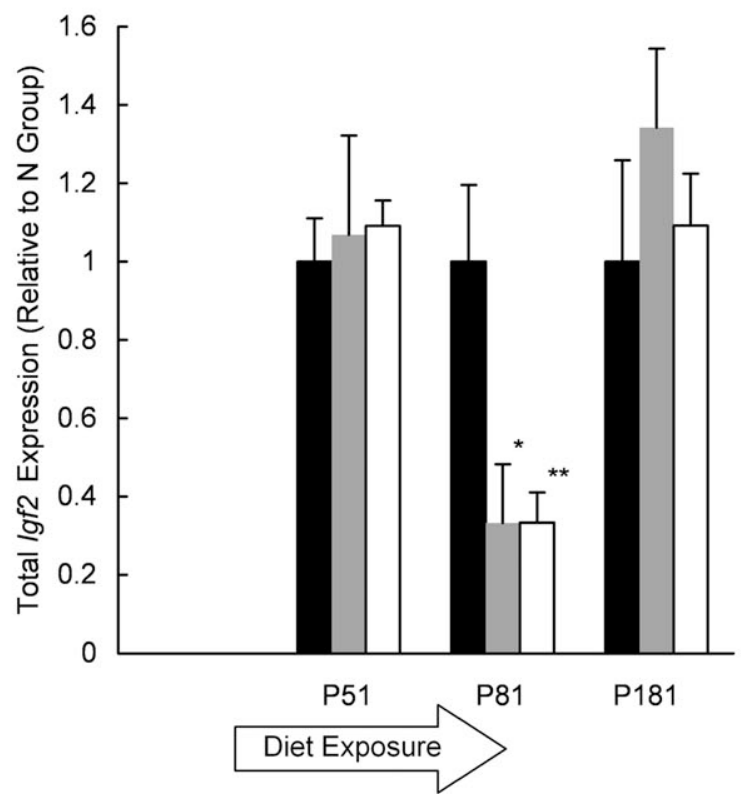

Figure 4. Effect of post-weaning diet on $\operatorname{Ig} f 2$ LOI and relative expression in kidney. (A) Igf2 LOI in kidney at different ages in mice fed different diets for 60 days post-weaning (mean \pm SEM of 4-5 animals per group at each age). Relative to animals weaned onto the $\mathrm{NC}$ diet, Igf2 LOI is increased in SC animals at 30 and 60 days post-weaning (i.e. P51 and P81), and in SD animals by 60 days post-weaning. Relative expression of maternal $\operatorname{Ig} f 2$ remains elevated in SD animals at $\mathrm{P} 181$, following the 100-day recuperation period during which all animals are provided NC diet. Animal sex did not affect Igf2 LOI in kidney, except at P181; data from males only are shown at P181. (B) Total Igf2 expression in kidney at different ages in mice fed different diets for 60-day post-weaning (mean \pm SEM of 4-5 animals per group at each age). At each age, Igf2 expression is related to that of the NC group. At P81, when both the SC and SD groups show significant LOI, both groups also show significant $70 \%$ reductions in $\operatorname{Ig} 2$ expression $\left({ }^{*} P<0.05,{ }^{* *} P<0.01,{ }^{* * *} P<0.001\right.$ relative to $\mathrm{NC}$ animals at same age). increase in relative maternal $\operatorname{Ig} 2$ expression, which persisted after the 100 days recuperation period (Fig. 4A).

Igf 2 LOI manifests as an increase in the ratio of maternal to paternal expression, which could result either from increased maternal expression or decreased paternal expression. To discriminate between these two possibilities, we used real-time polymerase chain reaction (PCR) to measure relative Igf2 expression in kidney at P51, P81 and P181 (i.e. 30, 60 and 160 days post-weaning). At 60 days post-weaning, $\operatorname{Ig} f 2$ expression in the SC and SD groups was only $30 \%$ that in the $\mathrm{NC}$ animals (Fig. 4B). These results clearly indicate that diet-induced LOI at 60 days post-weaning results not from de-repression of the maternal allele, but rather from a preferential repression of the paternal allele. No group difference in total $\operatorname{Ig} f 2$ expression was found after the 100-day recuperation period (P181).

Why did the SC diet induce Igf2 LOI? The SC diet formulation contains no nucleotides, whereas nucleotides are omnipresent in plant- and animal-based diets, including normal rodent chow. Although mammals can synthesize nucleotides de novo, they are considered a conditionally essential nutrient during early postnatal development when de novo synthesis may not meet requirements (27). Because the mammalian one-carbon metabolic pathways that provide methyl groups for DNA methylation reactions also contribute substrate for thymidilate and purine synthesis (24), we postulated that during the post-weaning period a dietary nucleotide deficiency might affect $I g f 2$ imprinting by impairing $\mathrm{CpG}$ methylation at Igf2 DMRs. To test this hypothesis, we weaned C57BL/Cast F1 mice onto the SC diet supplemented with yeast RNA as a source of nucleotides. At 60 days post-weaning, relative maternal expression of $I g f 2$ in kidney was identical to that of animals weaned onto the SC diet without RNA (data not shown), indicating that the $\operatorname{Igf} 2$ LOI caused by the SC diet is not attributable to dietary nucleotide deficiency.

Another obvious difference between the $\mathrm{NC}$ and SC diets is that the SC diet has a much higher sucrose content (see Supplementary Material). We, therefore, designed lowsucrose and high-sucrose natural ingredient diets to test the hypothesis that excessive sucrose intake during the postweaning period causes $I g f 2 \mathrm{LOI}$ in C57BL/Cast F1 mice. No group differences in $I g f 2 \mathrm{LOI}$ in kidney were found between C57BL/Cast F1 mice weaned onto either the low- or highsucrose diet (data not shown), indicating that the high-sucrose content of the SC diet is not responsible for its effect on $\operatorname{Ig} f 2 \mathrm{LOI}$.

\section{SC diet causes hypermethylation at Igf2 DMR2}

We next determined whether dietary methyl donor deficiency during the post-weaning period dysregulates $\operatorname{Ig} f 2$ allelic expression by hypomethylating the $H 19$ and Igf2 DMRs. To enable the reliable detection of subtle group differences in site-specific DNA methylation, we developed and validated a quantitative approach for direct bisulfite sequencing, in which post-bisulfite PCR products are sequenced manually and quantitated by phosphor imaging (Fig. 5). In kidney of animals at 60 days post-weaning, no group differences in total or allelespecific methylation were found in the H19 DMR or Igf2 DMR0 or DMR1 (data not shown). The SD diet likewise did not affect methylation at DMR2 (data not shown). Animal sex did not affect methylation in any genomic region. 
A

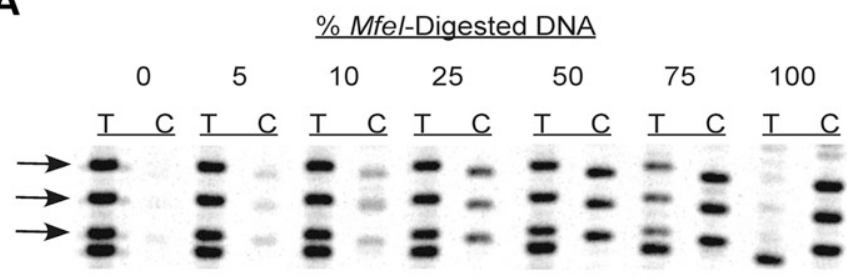

B

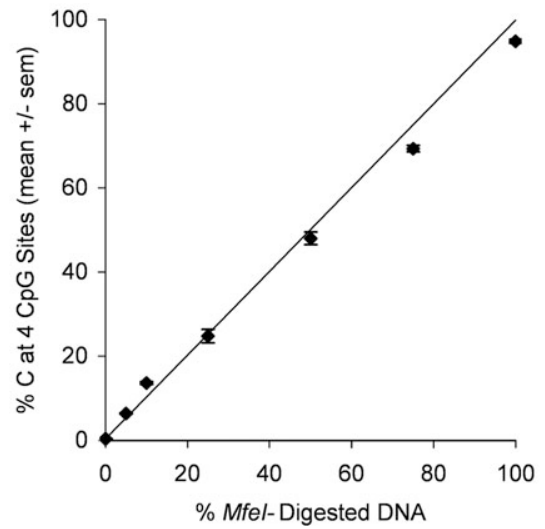

Figure 5. Validation of the direct-sequencing method for measurement of $\mathrm{CpG}$ methylation. (A) Gel image illustrating results of a mixing experiment in which H19 DMR methylation was quantitated in known quantities of MfeI- and SacI-digested C57BL/Cast DNA. MfeI digestion eliminates the maternally derived (unmethylated) allele, whereas SacI digestion eliminates the paternally derived (methylated) allele. The arrows show three $\mathrm{CpG}$ sites in the $H 19$ DMR. (B) Results of three independent mixing and sequencing experiments. The relationship between \% MfeI-digested DNA and \% C averaged over four $\mathrm{CpG}$ sites in the H19 DMR departs only slightly from the line of identity (shown) in the mixtures corresponding to 75 and $100 \%$ methylation. This is consistent with deamination of a small proportion of methylated $\mathrm{C}$ to $\mathrm{U}$ (i.e. overconversion). Proportional methylation as low as $5 \%$ of total is clearly detectable.

Compared with NC animals, those fed the SC diet had higher total DMR2 methylation levels at 30 days postweaning (Fig. 6B). At 60 days post-weaning, this group difference appeared quantitatively similar but failed to reach statistical significance (Fig. 6C). Analyzing the combined data at 30 and 60 days post-weaning, however, showed that relative to the $\mathrm{NC}$ diet, the $\mathrm{SC}$ diet induced highly significant $(P<0.01)$ hypermethylation at DMR2. We next employed an allele-specific PCR approach (Fig. 6A) to quantitate independently site-specific $\mathrm{CpG}$ methylation on the maternal and paternal alleles of DMR2. Group differences in paternalspecific DMR2 methylation mirrored those in total DMR2 methylation: analyzing the combined data at days 30 and 60 post-weaning demonstrated a highly significant $(P<0.01)$ hypermethylation of paternal DMR2 caused by the SC diet (Fig. 6B and C). Conversely, there were no group differences in maternal-specific DMR2 methylation (Fig. 6B and C). Hence, the DMR2 hypermethylation caused by the SC diet was specific to the paternal allele. Very interestingly, across all diet groups at 30 days post-weaning, Igf2 LOI was correlated with paternal DMR2 methylation (Fig. 6D). This relationship was not observed at 60 days post-weaning (Fig. 6D) or after the 100 days recuperation period.

We found no group differences in total or allele-specific DMR2 methylation in kidney DNA 100 days after the postweaning diet exposure (data not shown). Hence, these data demonstrate that diet-induced changes in $\operatorname{Ig} f 2$ allelic expression are maintained without persistent changes in methylation at any of the DMRs known to regulate Igf2 allelic expression. Nonetheless, it is intriguing that of all the Igf2 DMRs, only at DMR2 was the methylation state labile to post-weaning diet. To investigate why this might be, we examined the ontogeny of total and allele-specific methylation at the four Igf2 DMRs by comparing methylation state in E18 kidney with that at 60 days post-weaning. Total and allelespecific methylation at the H19 DMR, Igf2 DMR0 and DMR1 did not change over this period (data not shown), indicating that epigenotype at these genomic regions is fully established during prenatal kidney development. Conversely, total and allele-specific methylation at DMR2 showed substantial developmental changes during the postnatal period. From late gestation to adulthood, the monotonic increase in total DMR2 methylation reflected discrete periods of increasing methylation on the maternal and paternal alleles, respectively (Fig. 7).

Because the vast majority of $\mathrm{CpG}$ sites in mammalian genomes are within repetitive elements, bisulfite sequencing of repetitive elements can be used to estimate global DNA methylation (28). Hence, to determine if post-weaning diet caused changes in global DNA methylation, we assessed $\mathrm{CpG}$ methylation of generic intracisternal A particle (IAP) sequence in kidney genomic DNA from animals at 60 days post-weaning. At six $\mathrm{CpG}$ sites within generic IAP long-terminal repeat region sequence, average percent methylation was over $90 \%$ and did not differ among the diet groups (data not shown), indicating that the hypermethylation found at $\operatorname{Ig} 2 \mathrm{DMR} 2$ in SC animals occurred in the absence of a genome-wide increase in $\mathrm{CpG}$ methylation.

\section{Both the SC and SD diets affect one-carbon metabolism}

We also determined if the DMR2 hypermethylation caused by the SC diet was mediated by an induced alteration in transmethylation potential. $S$-adenosylmethionine (SAM) and $S$-adenosylhomocysteine (SAH) concentrations are important determinants of transmethylation potential because they are the direct methyl donor and byproduct of biological methylation reactions, respectively (24). Hepatic [SAM] and [SAH] at 60-day post-weaning were, therefore, measured by high-performance liquid chromatography (HPLC). As expected, relative to animals weaned onto the NC diet, the SD diet decreased [SAM] and increased $[\mathrm{SAH}]$, resulting in a 10 -fold decrease in the SAM:SAH 'methylation index' (Fig. 8). Interestingly, the SC diet caused both a non-significant decrease in [SAM] and nonsignificant increase in $[\mathrm{SAH}]$ which together resulted in a highly significant $60 \%$ decrease in the SAM:SAH methylation index (Fig. 8). 
A

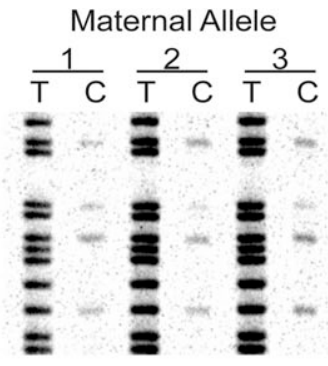

Paternal Allele

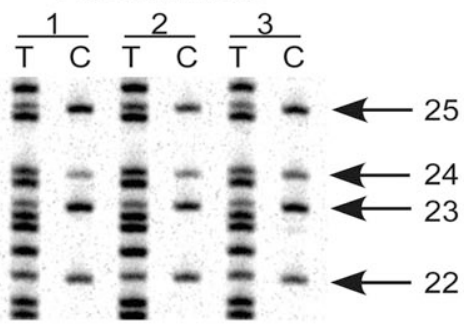

B

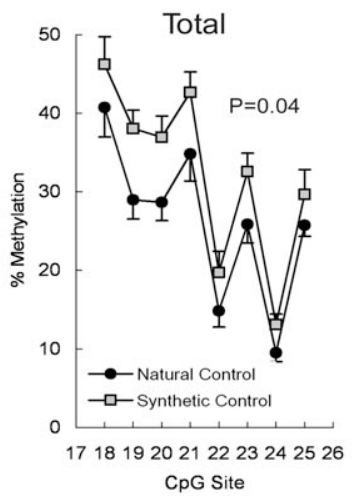

C

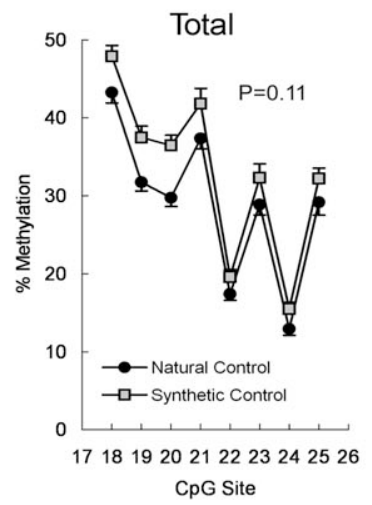

30 d Post-Weaning
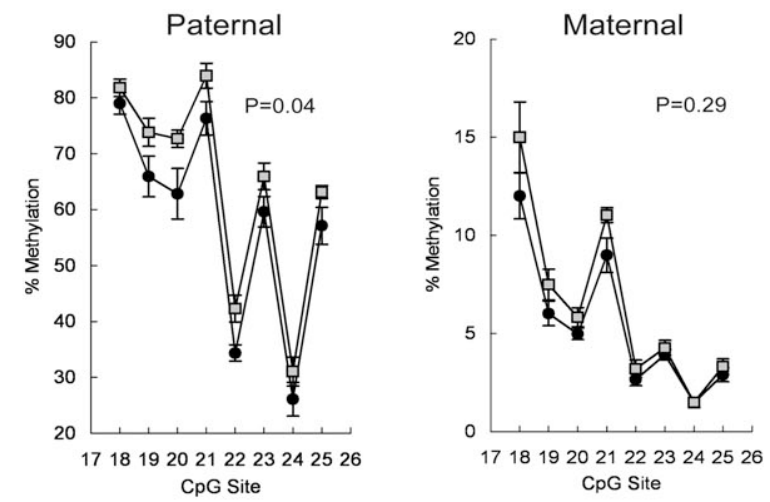

\section{$60 \mathrm{~d}$ Post-Weaning}

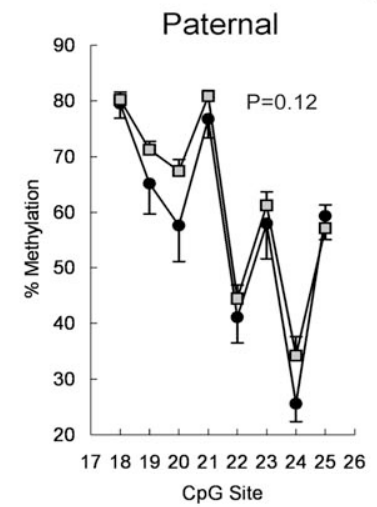

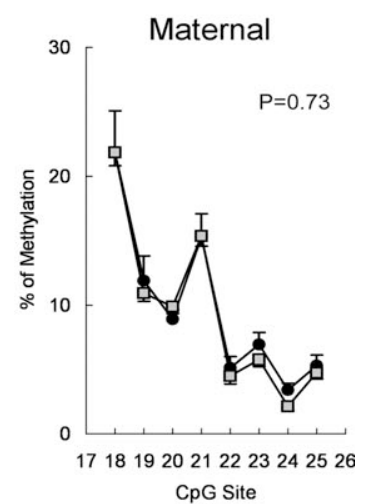

D

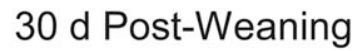

60 d Post-Weaning
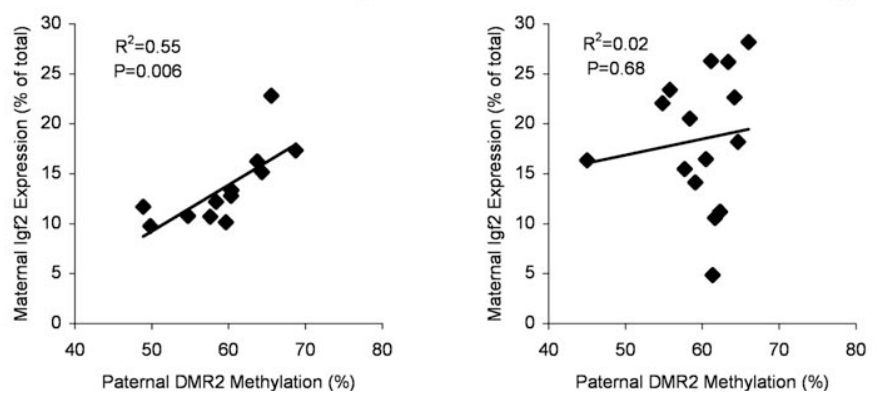

Figure 6. Effect of post-weaning diet on total and allele-specific methylation at Igf2 DMR2. (A) Gel image illustrating allele-specific bisulfite sequencing of CpG sites 22-25 of Igf2 DMR2 (46). Shown are maternal-specific and paternal-specific results from the same three C57BL/Cast F1 hybrid mice, demonstrating preferential methylation on the paternal allele. Total, paternal and maternal DMR2 CpG methylation are compared between NC and SC kidney at 30 (B) and 60 days postweaning $(\mathbf{C})$ (mean \pm SEM of 4-5 animals per group at each age). Analyzing the combined results at 30 and 60 days post-weaning shows that the SC diet causes a highly significant $(P<0.01)$ hypermethylation specific to the paternal allele. The SD diet did not affect DMR2 methylation. (D) Combining data from all the three diet groups shows that Igf 2 LOI is highly correlated $(P<0.01)$ with average paternal DMR2 methylation at 30 days but not at 60 days post-weaning. 


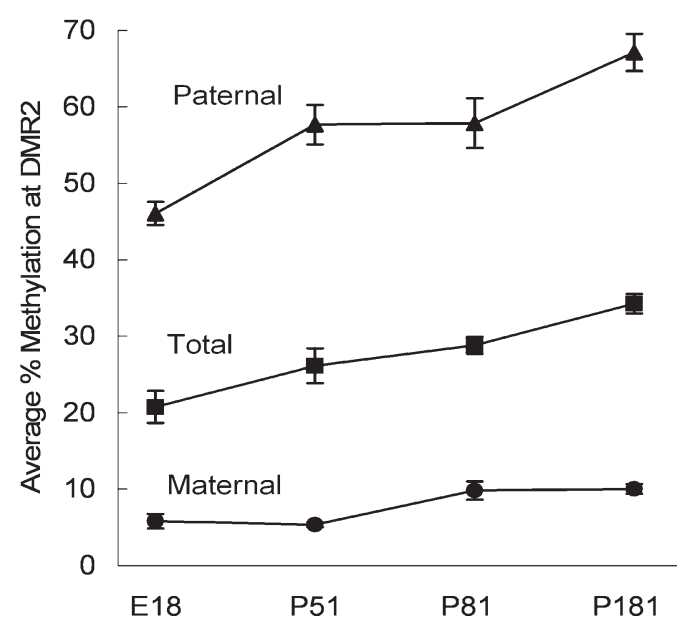

Figure 7. Ontogeny of total and allele-specific DMR2 methylation from late gestation to adulthood. Each point is the mean \pm SEM of average DMR2 methylation across sites 18-25 (46) of five mice. DMR2 methylation undergoes allele-specific changes during the postnatal period.

\section{DISCUSSION}

Several authors have proposed that nutrition and other environmental stimuli during development may induce persistent changes in the epigenetic regulation of genomically imprinted genes (17-19). This study is the first to actually demonstrate such a phenomenon in vivo. The media used to culture early mouse embryos can affect methylation and allelic expression of imprinted genes (20), but it is unknown whether such in vitro nutritional effects persist beyond fetal development. Hypomethylation and Igf2 LOI have been induced by injecting mice postnatally with the Dnmt1 inhibitor 5-azacytidine (29), and H19 LOI in hyperhomocysteinemic human adults can be ameliorated by oral folate therapy (30) but, likewise, the persistence of these effects has not been reported.

It is often assumed that $\operatorname{Ig} f 2 \mathrm{LOI}$ results from up-regulation of the normally 'silenced' maternal allele (31), which would cause a maximal 2-fold increase in total $\operatorname{Ig} 2$ expression. Most studies of IGF2 LOI, however, report only allelic expression ratios and provide no information on total IGF2 expression (6-8). Of course, an increase in the maternal: paternal expression ratio can result from either increased maternal expression or decreased paternal expression. Although Igf 2 overexpression frequently occurs in tumor tissue, it often shows no correlation with LOI, indicating that Igf2 overexpression in tumor tissue is independent of $\operatorname{Ig} f 2$ LOI (32). This study demonstrates that $I g f 2$ LOI can result from downregulation of the paternal allele, causing an overall decrease in Igf2 expression. Clearly, studies examining Igf2 LOI should not assume that an increase in the maternal:paternal expression ratio reflects up-regulation of the maternal allele. Only by measuring both the allelic expression ratio and total $\operatorname{Ig} f 2$ expression in the same samples can one draw inferences regarding the relationship between LOI and $I g f 2$ expression.

The methyl-deficient diet used in this study was intended to cause persistent alterations in $\operatorname{Ig} 22$ imprinting by inducing hypomethylation at Igf2 DMRs. Thus, it was unexpected that the substantial and persistent $I g f 2$ LOI caused by dietary methyl donor deficiency (Fig. 4A) occurred without major changes in site-specific $\mathrm{CpG}$ methylation at any of the four DMRs known to regulate Igf2 imprinting. Our findings prompted a critical evaluation of the literature examining the association between $H 19$ and $\operatorname{Ig} f 2$ allelic methylation and Igf2 LOI.

Although it is dogmatic that allele-specific CpG methylation is an important regulator of IGF2 imprinting, IGF2 LOI in humans comprises a heterogeneous class of phenomena showing inconsistent association with aberrant allelic methylation at any specific DMR. IGF2 LOI was first documented in Wilms' tumor (33), and in that childhood kidney cancer is correlated with bi-allelic methylation of the H19 DMR (15). However, approximately half of Wilms' tumor samples without IGF2 LOI also show bi-allelic methylation at the H19 DMR (15), demonstrating that $H 19$ hypermethylation alone is insufficient to cause $I G F 2$ LOI in Wilms' tumor. $I G F 2$ LOI in colorectal cancer does not correlate with biallelic methylation at the $H 19$ DMR. Rather, it is associated with loss of methylation at the human 'IGF2 DMR' that is orthologous to mouse DMR0 (16). Also contrary to the situation in Wilms' tumor, there is often loss of methylation at the $H 19$ DMR in colorectal cancer, but this is not correlated with IGF2 LOI (16). IGF2 LOI often occurs in Beckwith-Weidemann syndrome and is implicated in the somatic overgrowth and tumor predisposition seen in that developmental syndrome (9). Most cases of BeckwithWeidemann syndrome occur without any identifiable genetic lesion, and among those sporadic cases $I G F 2$ LOI usually occurs without detectable abnormalities in allelic methylation at the H19 or IGF2 DMRs (14). Hence, BeckwithWeidemann syndrome demonstrates clearly that $I G F 2$ LOI can occur independent of allelic methylation changes.

All these data, together with the results of the current study, demonstrate that substantial changes in allelic expression of $I G F 2$ can occur without appreciable changes in DNA methylation at known DMRs. The mouse model described here may, therefore, yield insights into the molecular mechanisms of Igf2 LOI. For example, specific histone modifications may play a primary role in mediating diet-induced changes in allelic expression. Future studies will be required to elucidate these alterations, with the ultimate goal of determining if similar mechanisms underlie and unify the seemingly heterogeneous phenomena of IGF2 LOI in humans.

This study demonstrates the exquisite sensitivity with which site-specific $\mathrm{CpG}$ methylation can be measured by direct bisulfite sequencing and phosphor-imager quantitation (25), compared with the more traditional method of sequencing a sample of cloned PCR products (34). Because of the errors inherent in sampling from a population of clones, detecting small group differences in methylation by that method would require an inordinate number of clones to be sequenced for each animal studied. Conversely, by the direct sequencing approach described here, the $10-30 \%$ site-specific hypermethylation in the DMR2 region of SC animals (Fig. 6) was detectable. Our ability to detect such small group differences illustrates the level of confidence with which we were able to rule out group differences in allelic methylation in the other DMRs. Comparing the results of the direct sequencing 

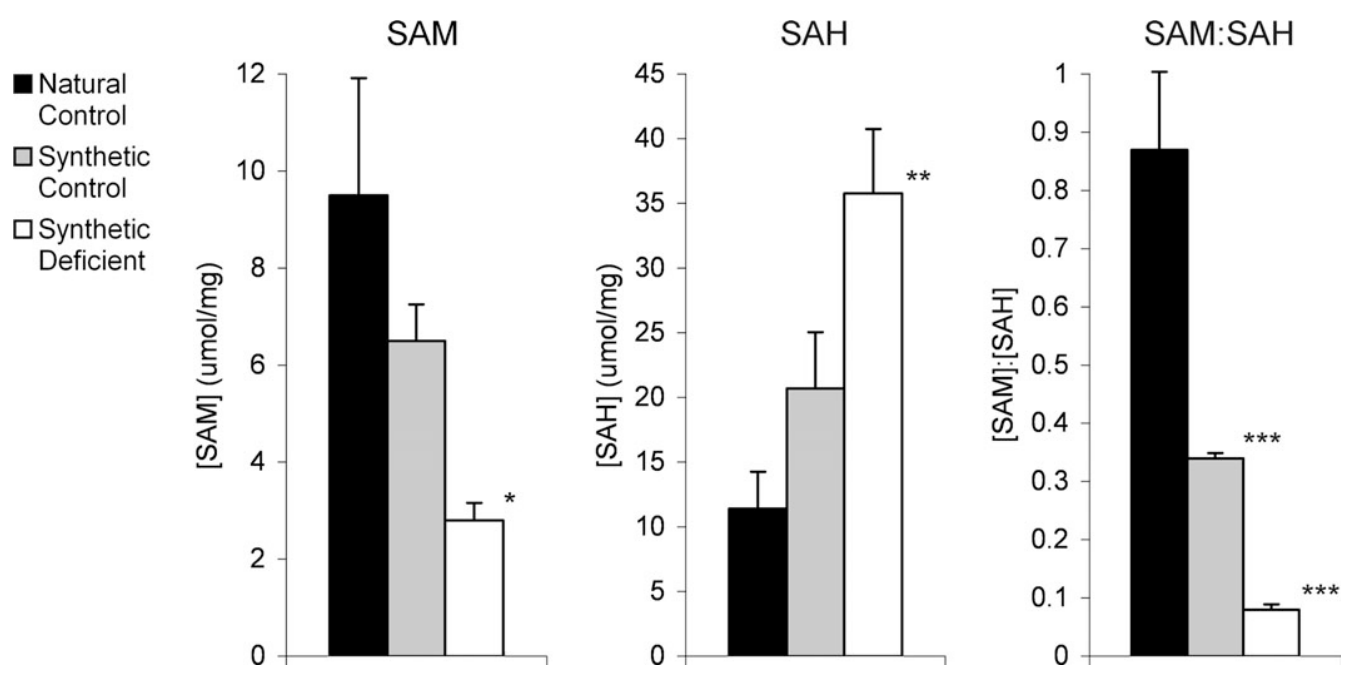

Figure 8. SAM, SAH and the SAM:SAH ratio in liver of animals weaned onto different diets for 60 days (mean \pm SEM of five animals/group). Both the SC and SD diet cause significant decreases in the SAM:SAH methylation index $\left({ }^{*} P<0.05,{ }^{* *} P<0.01,{ }^{* * *} P<0.001\right.$ relative to $\mathrm{NC}$ animals).

approach with the best published example of bisulfite sequencing of DMR2 in mouse kidney using the clone-sampling approach (34) demonstrates that our method yields quantitatively similar (though more precise) results. Strikingly, the 'w' pattern caused by site-to-site variation from sites 21-25 (Fig. 6) replicates that found in the earlier study (34).

We postulated that as cellular proliferation continues in the kidney during the post-weaning period in the mouse (35), deficiency of dietary methyl donors and cofactors during this period might cause a failure to maintain established patterns of methylation during DNA replication. But our results demonstrate that it is very difficult to induce hypomethylation at Igf2 DMRs in kidney DNA by dietary methyl donor deficiency during this period. It was surprising that the SC diet, which reduced the SAM:SAH methylation index, caused hypermethylation at DMR2. This result, however, is actually consistent with previous studies showing locus-specific hypermethylation in the face of diet-induced reductions in the SAM:SAH ratio $(36,37)$. Future studies will be needed to address the important issue of specifically what is lacking (or present) in the SC diet that causes Igf2 LOI and DMR2 hypermethylation. This question is especially important in the context of infant nutrition: just as the synthetic SC diet used here was previously thought to be an adequate substitute for natural ingredient diets, infant formulas are essentially semi-synthetic substitutes for human milk. It is, therefore, possible that persistent differences observed between formulafed and human milk-fed individuals (38) are the result of epigenetic alterations induced by subtle nutritional differences between human milk and infant formula.

Of the four murine DMRs known to regulate Igf2 allelic expression, only at DMR2 was methylation responsive to post-weaning diet. Examining the postnatal ontogeny of allelic methylation provided a potential explanation why. Unlike the H19 DMR and Igf2 DMR0 and DMR1, methylation at DMR2 demonstrates developmental changes throughout the postnatal period and into adulthood (Fig. 7). Our findings, therefore, support the hypothesis that environmental perturbation of epigenotype occurs most readily at loci at which epigenotype is undergoing developmental change (19). Recent studies in mice with engineered deletions of Igf2 DMRs provide additional insight into why the methylation state at DMR2 may be especially labile. Maintenance of the methylation state at DMR2 depends upon interactions with other Igf2 and $H 19$ DMRs; mice carrying a deletion of either the H19 DMR or Igf2 DMR1 show aberrant methylation at DMR2, whereas deletion of DMR2 does not reciprocally affect the other DMRs (11). Dietary perturbation might, therefore, interfere with the chromatin secondary structure postulated to facilitate interactions among the Igf2 DMRs (11), preferentially causing epigenetic instability at DMR2.

Our results, showing an environmental influence on $\operatorname{Ig} f 2$ LOI, may at first appear contradictory to a recent human epidemiologic study which concluded that IGF2 LOI occurs independent of environmental factors such as diet and smoking (8). That study, however, looked for correlations between IGF2 imprinting status and environmental influences in adulthood. It did not evaluate the possibility that diet and other environmental influences during development could induce persistent IGF2 LOI (39). Our results demonstrate that nutrition during postnatal development can have a permanent effect on the epigenetic regulation of imprinted genes. It is, therefore, possible that such epigenetically based developmental plasticity (40) comprises a mechanistic link between early nutrition and human health.

\section{MATERIALS AND METHODS}

\section{Animals and diets}

C57BL/6J females were mated with Cast/EiJ males to generate F1 hybrid offspring with polymorphisms within coding and regulatory regions of $\operatorname{Ig} f 2$ (26). At the age of 21 days, offspring were weaned and assigned randomly to one of the three diets: (SD) a synthetic methyl-donor-deficient diet devoid of methionine, folic acid, vitamin $\mathrm{B}_{12}$ and choline 
(Harlan-Teklad, TD00605) [The essential amino-acid methionine was replaced by the homocysteine dimer homocystine (41).], (SC) a synthetic control diet (the synthetic diet formulation containing normal levels of methionine, folic acid, vitamin $\mathrm{B}_{12}$ and choline; Harlan-Teklad, TD99366) or (NC) a natural ingredient diet (LabDiet 5021 rodent diet). For the RNA supplementation experiment, the SC diet was supplemented with $2.5 \mathrm{~g} / \mathrm{kg}$ Torula yeast RNA (Sigma) (complete diet formulation is available in Supplementary Material). Mice were provided their respective diets for 60 days post-weaning. Tissues were collected from a subset of mice at 30 and 60 days post-weaning. After 60 days on the respective diets, all animals were placed on the natural ingredient diet for an additional 100 days (recuperation period) to determine if any epigenetic alterations induced by the experimental diets were maintained following a return to normal diet. All animal protocols were approved by the Institutional Animal Care and Use Committee at Duke University Medical Center and Baylor College of Medicine. Data on animal weight were analyzed by ANOVA (SAS GLM procedure).

\section{Measurement of $\operatorname{Ig} f \mathbf{2}$ expression}

To measure $I g f 2$ allelic expression, total RNA was isolated (RNA Stat-60, Tel-Test, Inc.), treated with DNase-I (Invitrogen), reverse-transcribed and PCR-amplified (SuperScript One-Step RT-PCR kit, Invitrogen) using primers Igf2-F (5'-GACGTGTCTACCTCTCAGGCCGTACTT-3') and Igf2-R (5'-GGGTGTCAATTGGGTTGTTTAGAGCCA-3') (40 cycles in total). The RT step utilized a gene-specific primer (Igf2-R). The $492 \mathrm{bp} \mathrm{RT}-\mathrm{PCR}$ products were gel-purified (GenElute Minus EtBr Column, Sigma); the $735 \mathrm{bp}$ products which would result from the amplification of genomic DNA were never observed. The purified Igf 2 cDNA was sequenced manually (ThermoSequenase radiolabeled terminator cycle sequencing kit; USB Corp.) using sequencing primer Igf2-S (5'-CCTCCCCACATCAGGCTG-3'), which anneals just upstream of a G/A SNP within Igf2 exon 6 (26). The sequencing reaction products were resolved by polyacrylamide gel electrophoresis and quantitated by phosphor-imaging (Molecular Dynamics Storm). The relative intensity of the $G$ and A bands at the interrogated SNP in the sequenced RT-PCR products provided a direct quantitation of the percentage of Igf2 expression derived from the maternally inherited allele (Fig. 3). To validate the direct sequencing method for quantitation of allelic expression, genomic DNA from C57BL and Cast mice was PCR-amplified using primers Igf2-F and Igf2-R. PCR products were gel-purified and quantitated spectrophotometrically. Known mixtures of the C57BL and Cast PCR products were prepared, manually sequenced and quantitated as described earlier. Data were analyzed by ANOVA (SAS GLM procedure).

To compare total Igf2 gene expression between groups, total RNA was isolated (RNA Stat-60) and reverse-transcribed (Taqman RT Reagents, Applied Biosystems) using random hexamer priming, according to the manufacturer's instructions. Igf2 expression was measured relative to a $\beta$-actin endogenous control by real-time PCR using FAM-labeled (FAM: 6-carboxy-fluoroscein) probes (Taqman Gene Expression Assays, Applied Biosystems) and a 7700 Sequence Detection
System (Applied Biosystems). Assays were run in triplicate. Non-RT-negative controls were run for every sample; these never reached detection threshold. $\beta$-Actin cycles to threshold $\left(C_{\mathrm{T}}\right)$ did not differ between diet groups at any age. Relative Igf2 expression was calculated as $2^{-\Delta \Delta C_{\mathrm{T}}}$ according to the manufacturer's instructions. For each sample, $\Delta C_{\mathrm{T}}=\operatorname{Igf2}$ $C_{\mathrm{T}}-\beta$-actin $\quad C_{\mathrm{T}}$ and $\Delta \Delta C_{\mathrm{T}}=$ sample $\Delta C_{\mathrm{T}}$ - reference group (NC) mean $\Delta C_{\mathrm{T}}$.

\section{Measurement of total and allele-specific $\mathrm{CpG}$ methylation}

Within each of the four DMRs known to regulate Igf2 allelic expression (11), 1-2 kb was sequenced from genomic DNA isolated from two C57BL and two Cast animals. Regions of identical sequence in both animals of the same genotype were used to assemble strain-specific contigs within each DMR. Strain-specific DMR sequence data were submitted to GenBank and assigned accession numbers as follows: H19 DMR C57BL no. AY849916, Cast no. AY849917; DMR0 C57BL no. AY849920, Cast no. AY849921; DMR1 C57BL no. AY849918, Cast no. AY849919; DMR2 C57BL no. AY849922, Cast no. AY849923.

Site-specific $\mathrm{CpG}$ methylation was measured by bisulfite sequencing and direct phosphor-imager quantitation (25). (Treatment of DNA with sodium bisulfite deaminates cytosine to uracil; 5-methyl-cytosine is protected from deamination.) Briefly, $2 \mu \mathrm{g}$ of genomic DNA (42) was denatured in $50 \mu \mathrm{l}$ of $0.3 \mathrm{M} \mathrm{NaOH}\left(20 \mathrm{~min}, 37^{\circ} \mathrm{C}\right)$ and deamination was initiated by adding $450 \mu \mathrm{l}$ of a solution of saturated sodium bisulfite (Sigma) and $10 \mathrm{~mm}$ hydroquinone (Sigma), $\mathrm{pH}$ 5.0. Samples were overlaid with mineral oil and incubated for $3 \mathrm{~h}$ at $55^{\circ} \mathrm{C}$ in the dark. Samples were then desalted (Wizard DNA Cleanup System, Promega), eluted in $50 \mu 11 \mathrm{~mm}$ Tris-Cl, $\mathrm{pH} 8.0$, and desulfonated by addition of $5.5 \mu \mathrm{l} 3 \mathrm{M} \mathrm{NaOH}$ and incubation at $37^{\circ} \mathrm{C}$ for $20 \mathrm{~min}$. Bisulfite-modified DNA was ethanol-precipitated, washed and suspended in $20 \mu \mathrm{l}$ $1 \mathrm{~mm}$ Tris-Cl, pH 8.0. Genomic regions of interest were PCR-amplified; each reaction (40 cycles) employed $4 \mu \mathrm{l}$ bisulfite-modified DNA in a $50 \mu$ l reaction volume, using Platinum Taq DNA polymerase (Invitrogen). Bands were gel-purified (GenElute Minus EtBr spin columns, Sigma) and sequenced manually (ThermoSequenase radiolabeled terminator cycle sequencing kit; USB Corp.), $T_{\text {anneal }}=$ primer $T_{\text {melt }}, 35$ cycles. Sequencing products were resolved by polyacrylamide gel electrophoresis, with a blank lane between the $C$ and $T$ lanes to avoid signal overlap. Percent methylation at each $\mathrm{CpG}$ site was quantitated by phosphor imaging $\left(\%\right.$ methylation $\left.=100 \times\left[C_{\text {volume }} /\left(C_{\text {volume }}+T_{\text {volume }}\right)\right]\right)(25)$.

In the H19 DMR, a C57BL-specific MfeI restriction site and a Cast-specific $\mathrm{SacI}$ restriction site were identified. To assess paternal (Cast)-specific methylation at the H19 DMR of the C57BL/Cast F1 animals, $2 \mu \mathrm{g}$ of genomic DNA was digested overnight with $20 \mathrm{U}$ of $M f e I$, ethanol-precipitated and washed before bisulfite treatment. Maternal-specific methylation at the H19 DMR was assessed after digesting genomic DNA with SacI. Sequencing confirmed that digestion was complete. Total H19 DMR methylation was assessed in undigested DNA. Following bisulfite modification, hypersensitive region 2 (HS2) within the H19 DMR was PCR-amplified using primers HS2BF2 (5'-AGGGTTGTAAATAATTTTGAAATTG-3') and 
HS2BR2 (5'-ATTCAATATATAAAAAAACCATTCC-3'). The $748 \mathrm{bp}$ PCR products were gel-purified and sequenced as described earlier using sequencing primer HS2BF4 (5'-ATAGAAGTTGTTATGTGTAATAAGGG-3'). This approach enabled quantitation of percent methylation at five CpG sites overlapping the most $5^{\prime}$ CTCF site in HS2 (nt. 1465-1494 of GenBank accession no. AY849916).

In DMR0, a C57BL-specific BanI restriction site and a Castspecific EcoNI were identified. Hence, paternal- and maternalspecific methylation at DMR0 were assessed in genomic DNA digested with BanI or EcoNI, respectively, prior to bisulfite sequencing. DMR0 fragments (179 bp) were PCR-amplified from BanI-digested, bisulfite-modified DNA using primers DMR0BF4 (5'-GGAGAGTAGAAGTTATTTTTAGGGG-3') and DMR0BR1 (5'-CCCTCCAAAACAAAAAATACTC-3'). DMR0 fragments ( $451 \mathrm{bp})$ were PCR-amplified from EcoNIdigested, bisulfite-modified DNA using primers DMR0BF2 (5'ATTTTTGTATATAGTTTTTATTTTTTAGTTAATAGG-3') and DMR0BR1. PCR products were gel-purified and manually sequenced using sequencing primer DMR0BF5 (5'-AGTT ATTTTTAGGGGTTTTGTTATTATG-3'). Percent methylation was quantitated at eight $\mathrm{CpG}$ sites within DMR0 (nt. $786-874$ of GenBank accession no. AY849920) 100 bp 3' of the $\mathrm{P}_{0}$ transcription start site (43).

In the DMR1 region, a Cast-specific TspRI site was identified, but no C57BL-specific restriction sites were found. Maternalspecific methylation at DMR1 was assessed in genomic DNA digested with $T_{s} p$ RI prior to bisulfite modification. Sequencing confirmed that digestion was complete. DMR1 fragments (265 bp) were PCR-amplified using primers DMR1BF1 (5'-GAGTTATATTTTGATTAAATAAGGTT AGGTG-3') and DMR1BR1 (5'-TCACAACCTATAACC TATTTCAAAACC- $\left.3^{\prime}\right)$. PCR products were gel-purified and manually sequenced using sequencing primer DMR1BF3 (5'TAGAGgAagaAgaAtatATGTATATTTTGG-3'). Percent methylation was quantitated at four $\mathrm{CpG}$ sites (nt. 501-602 of GenBank accession no. AY849918) in the region of DMR1 overlapping HpaII site 3 as annotated by Eden et al. (44). Total DMR1 methylation was assessed in undigested DNA, and paternal methylation was calculated from the maternal and total values.

None of the single-nucleotide polymorphisms (SNPs) identified within $1000 \mathrm{bp}$ of DMR2 were located within restriction enzyme recognition sites. Instead of allele-specific digestion, we therefore employed allele-specific PCR to study separately CpG methylation on the maternal and paternal alleles at DMR2. We identified a G/A SNP approximately 300 bp 3' of DMR2 and designed allele-specific primers terminating at the SNP. Secondary or tertiary internal mismatches were included in the primers (45) to enhance discrimination between the C57BL and Cast alleles. To assess maternalspecific DMR2 methylation in C57BL/Cast F1 hybrids, DMR2 fragments (519 bp) were first PCR-amplified from bisulfite-modified genomic DNA with generic primer DMR2BF4 (5'-GGGAGTTTAGGTTAATATGATATTTT G-3') and C57BL-specific primer DMR2BBR (5'-ACCAATC AAATTTAATTTTTTAAACC- $\left.3^{\prime}\right), T_{\text {anneal }}=52.6^{\circ} \mathrm{C}$. Paternalspecific methylation was assessed by first PCR amplifying DMR2 fragments (521 bp) from bisulfite-modified genomic DNA using primer DMR2BF4 and Cast-specific primer DMR2CBR2 (5'-AAACCAATCAAATTTAATTTTTTAAC
AT- $\left.3^{\prime}\right), T_{\text {anneal }}=57^{\circ} \mathrm{C}$. Total DMR2 methylation was quantitated by amplifying the DMR2 region ( $360 \mathrm{bp}$ ) from bisulfitemodified DNA using generic primers DMR2BF4 and DMR2BR1 (5'-ACTATCCCTACTCAAAAAAAAATCAC-3'). In all cases, the PCR products were gel-purified, sequenced manually using sequencing primer DMR2BF6 (5'-GGGTAA GTTTTTTTAATATGATATTTGG-3') and site-specific CpG methylation quantitated by phosphor-imaging. This approach enabled measurement of percent methylation at the eight sites comprising core DMR2 [sites 18-25 as annotated by Murrell et al. (46); nt 1227-1280 of GenBank accession no. AY849922].

To validate our direct-sequencing approach for quantitating site-specific $\mathrm{CpG}$ methylation, we took advantage of the naturally occurring 0 and $100 \%$ methylation status of the H19 DMR on the maternal and paternal alleles, respectively, in C57BL/Cast F1 mice. C57BL/Cast genomic DNA was quantitated and digested (10-fold overdigestion, overnight) with $\mathrm{MfeI}$ or $\mathrm{SacI}$ to cleave the maternal (unmethylated) or paternal (methylated) allele, respectively. Digested DNA was EtOHprecipitated and resuspended. To balance potential losses due to incomplete recovery, four separate digests were conducted with each enzyme and the samples were pooled following EtOH precipitation. Known mixtures of MfeI- and SacI-digested DNA were prepared, and bisulfite modification and sequencing of the H19 DMR region were conducted as described earlier.

Site-specific CpG methylation at generic IAP long-terminal repeat region sequence throughout the mouse genome was measured by bisulfite sequencing. Primer design was based on IAP consensus sequence (RepBase accession no. IAPLTR1a_MM) to ensure that a broad range of IAPs were interrogated. Bisulfite-modified kidney genomic DNA was PCR-amplified using primers IAPLTR F2 (5'-GTGG TTTTTTATTTTATGTGTTTTG-3') and IAPLTR R1 (5'-CACCACAAACCAAAATCTTC-3'). PCR products were gel-purified and manually sequenced using sequencing primer IAPLTR F2 and quantitated by phosphor-imaging. This approach enabled assessment of percent methylation at six $\mathrm{CpG}$ sites within generic IAP long-terminal repeat sequence (nt. 124-180 of RepBase accession no. IAPLTR1a_MM). DMR and IAP methylation data were analyzed by ANOVA (SAS mixed model).

\section{HPLC determination of SAM and SAH}

Hepatic concentrations of SAM and SAH were measured using the method of Herbig et al. (47). Approximately $50 \mathrm{mg}$ of liver was weighed and homogenized in $500 \mu \mathrm{l}$ $0.1 \mathrm{M} \mathrm{NaAcO}(\mathrm{pH} 5.5)$ and cellular proteins were precipitated by addition of $312 \mu 1 \quad 10 \%$ perchloric acid. After centrifugation $\left(2000 \mathrm{~g}, 10 \mathrm{~min}, 4^{\circ} \mathrm{C}\right)$, each supernatant was transferred to a clean tube, neutralized by addition of $140 \mu 11 \mathrm{M} \mathrm{NaPO}_{4}$ (pH 11.5) and diluted with $1 \mathrm{ml}$ deionized $\mathrm{H}_{2} \mathrm{O}$. Each sample was then applied to a C18 Sep-Pak cartridge (Waters Corp.) primed with $5 \mathrm{~mm}$ 1-heptanesulfonic acid (Alfa Aesar) in methanol. The cartridges were then washed with $5 \mathrm{ml}$ deionized $\mathrm{H}_{2} \mathrm{O}$ before SAM and $\mathrm{SAH}$ were eluted in $2 \mathrm{ml}$ of methanol. Fifty microliters of $3 \mathrm{M} \mathrm{NaAcO}$ was added to each eluate before drying under vacuum at ambient 
temperature overnight. SAM and SAH were re-suspended in $250 \mu \mathrm{l} \mathrm{H}_{2} \mathrm{O}$ and converted to their fluorescent derivatives by the addition of $50 \mu \mathrm{l}$ chloroacetylaldehyde (50 wt.\%, SigmaAldrich) and incubation at $60^{\circ} \mathrm{C}$ for $1 \mathrm{~h}$. Purified SAM (New England Biolabs) and SAH (Sigma-Aldrich) were quantitated spectrophotometrically and used to generate standard curves. The standards were prepared using the same complete protocol as described for the tissue extracts. Samples and standards were loaded onto a C 8 column $(5 \mu \mathrm{m}, 250 \times 4.6 \mathrm{~mm}$; Phenomenex) and solvent delivery was performed by two Shimadzu LC-10ADVP pumps controlled by a SCL-10AVP system controller. A two-buffer system was used to separate SAM and SAH, as described by Herbig et al. (47). SAM and SAH were detected with a Shimadzu RF-10AXL spectrofluorometric detector $\left(\lambda_{\mathrm{ex}}=270 \mathrm{~nm}\right.$ and $\left.\lambda_{\mathrm{em}}=410 \mathrm{~nm}\right)$. Data were analyzed by ANOVA (SAS GLM procedure).

\section{SUPPLEMENTARY MATERIAL}

Supplementary Material is available at HMG Online.

\section{ACKNOWLEDGEMENTS}

We gratefully acknowledge the helpful comments of Qiang Tong and Ignatia Van den Veyver. This work was supported by a fellowship from the Dannon Institute, NIH grants DK063781 and DK070007 and USDA CRIS 6250-51000-049 (R.A.W.) and NIH grants CA25951, ES13053 and ES08823 (R.L.J.).

Conflict of Interest statement. None declared.

\section{REFERENCES}

1. Jaenisch, R. and Bird, A. (2003) Epigenetic regulation of gene expression: how the genome integrates intrinsic and environmental signals. Nat. Genet., 33 (suppl.), 245-254.

2. Petronis, A. (2001) Human morbid genetics revisited: relevance of epigenetics. Trends Genet., 17, 142-146.

3. Jiang, Y.H., Bressler, J. and Beaudet, A.L. (2004) Epigenetics and human disease. Annu. Rev. Genom. Hum. Genet., 5, 479-510.

4. Egger, G., Liang, G., Aparicio, A. and Jones, P.A. (2004) Epigenetics in human disease and prospects for epigenetic therapy. Nature, 429, 457-463.

5. Falls, J.G., Pulford, D.J., Wylie, A.A. and Jirtle, R.L. (1999) Genomic imprinting: implications for human disease. Am. J. Pathol., 154, 635-647.

6. Sakatani, T., Wei, M., Katoh, M., Okita, C., Wada, D., Mitsuya, K., Meguro, M., Ikeguchi, M., Ito, H., Tycko, B. et al. (2001) Epigenetic heterogeneity at imprinted loci in normal populations. Biochem. Biophys Res. Commun., 283, 1124-1130.

7. Cui, H., Cruz-Correa, M., Giardiello, F.M., Hutcheon, D.F., Kafonek, D.R., Brandenburg, S., Wu, Y., He, X., Powe, N.R. and Feinberg, A.P. (2003) Loss of IGF2 imprinting: a potential marker of colorectal cancer risk. Science, 299, 1753-1755.

8. Cruz-Correa, M., Cui, H., Giardiello, F.M., Powe, N.R., Hylind, L., Robinson, A., Hutcheon, D.F., Kafonek, D.R., Brandenburg, S., Wu, Y. et al. (2004) Loss of imprinting of insulin growth factor II gene: a potential heritable biomarker for colon neoplasia predisposition. Gastroenterology, 126, 964-970.

9. Weksberg, R., Smith, A.C., Squire, J. and Sadowski, P. (2003) BeckwithWiedemann syndrome demonstrates a role for epigenetic control of normal development. Hum. Mol. Genet., 12 (Spec No. 1), R61-R68.

10. Sandovici, I., Leppert, M., Hawk, P.R., Suarez, A., Linares, Y. and Sapienza, C. (2003) Familial aggregation of abnormal methylation of parental alleles at the IGF2/H19 and IGF2R differentially methylated regions. Hum. Mol. Genet., 12, 1569-1578.
11. Lopes, S., Lewis, A., Hajkova, P., Dean, W., Oswald, J., Forne, T., Murrell, A., Constancia, M., Bartolomei, M., Walter, J. et al. (2003) Epigenetic modifications in an imprinting cluster are controlled by a hierarchy of DMRs suggesting long-range chromatin interactions. Hum. Mol. Genet., 12, 295-305.

12. Vu, T.H., Li, T., Nguyen, D., Nguyen, B.T., Yao, X.M., Hu, J.F. and Hoffman, A.R. (2000) Symmetric and asymmetric DNA methylation in the human IGF2-H19 imprinted region. Genomics, 64, 132-143.

13. Sullivan, M.J., Taniguchi, T., Jhee, A., Kerr, N. and Reeve, A.E. (1999) Relaxation of IGF2 imprinting in Wilm's tumours associated with specific changes in IGF2 methylation. Oncogene, 18, 7527-7534.

14. Reik, W., Brown, K.W., Schneid, H., Le Bouc, Y., Bickmore, W. and Maher, E.R. (1995) Imprinting mutations in the Beckwith-Wiedemann syndrome suggested by altered imprinting pattern in the IGF2-H19 domain. Hum. Mol. Genet., 4, 2379-2385.

15. Cui, H., Niemitz, E.L., Ravenel, J.D., Onyango, P., Brandenburg, S.A., Lobanenkov, V.V. and Feinberg, A.P. (2001) Loss of imprinting of insulin-like growth factor-II in Wilms' tumor commonly involves altered methylation but not mutations of CTCF or its binding site. Cancer Res., 61, 4947-4950.

16. Cui, H., Onyango, P., Brandenburg, S., Wu, Y., Hsieh, C.L. and Feinberg, A.P. (2002) Loss of imprinting in colorectal cancer linked to hypomethylation of H19 and IGF2. Cancer Res., 62, 6442-6446.

17. Pembrey, M. (1996) Imprinting and transgenerational modulation of gene expression; human growth as a model. Acta Genet. Med. Gemellol. (Roma), 45, 111-125.

18. Thompson, S.L., Konfortova, G., Gregory, R.I., Reik, W., Dean, W. and Feil, R. (2001) Environmental effects on genomic imprinting in mammals. Toxicol. Lett., 120, 143-150.

19. Waterland, R.A. and Jirtle, R.L. (2004) Early nutrition, epigenetic changes at transposons and imprinted genes, and enhanced susceptibility to adult chronic diseases. Nutrition, 20, 63-68.

20. Khosla, S., Dean, W., Brown, D., Reik, W. and Feil, R. (2001) Culture of preimplantation mouse embryos affects fetal development and the expression of imprinted genes. Biol. Reprod., 64, 918-926.

21. Biniszkiewicz, D., Gribnau, J., Ramsahoye, B., Gaudet, F., Eggan, K., Humpherys, D., Mastrangelo, M.A., Jun, Z., Walter, J. and Jaenisch, R. (2002) Dnmt1 overexpression causes genomic hypermethylation, loss of imprinting, and embryonic lethality. Mol. Cell Biol., 22, 2124-2135.

22. Niemitz, E.L. and Feinberg, A.P. (2004) Epigenetics and assisted reproductive technology: a call for investigation. Am. J. Hum. Genet., 74, 599-609.

23. Waterland, R.A. and Garza, C. (1999) Potential mechanisms of metabolic imprinting that lead to chronic disease. Am. J. Clin. Nutr., 69, 179-197.

24. Van den Veyver, I. (2002) Genetic effects of methylation diets. Annu. Rev. Nutr., 22, 255-282.

25. Waterland, R.A. and Jirtle, R.L. (2003) Transposable elements: targets for early nutritional effects on epigenetic gene regulation. Mol. Cell. Biol., 23, 5293-5300.

26. Szabo, P.E. and Mann, J.R. (1995) Biallelic expression of imprinted genes in the mouse germ line: implications for erasure, establishment, and mechanisms of genomic imprinting. Genes. Dev., 9, 1857-1868.

27. Aggett, P., Leach, J.L., Rueda, R. and MacLean, W.C., Jr. (2003) Innovation in infant formula development: a reassessment of ribonucleotides in 2002. Nutrition, 19, 375-384.

28. Yang, A.S., Estecio, M.R., Doshi, K., Kondo, Y., Tajara, E.H. and Issa, J.P. (2004) A simple method for estimating global DNA methylation using bisulfite PCR of repetitive DNA elements. Nucleic Acids Res., 32, e38.

29. Hu, J.F., Nguyen, P.H., Pham, N.V., Vu, T.H. and Hoffman, A.R. (1997) Modulation of Igf2 genomic imprinting in mice induced by 5-azacytidine, an inhibitor of DNA methylation. Mol. Endocrinol., 11, 1891- 1898

30. Ingrosso, D., Cimmino, A., Perna, A.F., Masella, L., De Santo, N.G., De Bonis, M.L., Vacca, M., D’Esposito, M., D’Urso, M., Galletti, P. et al. (2003) Folate treatment and unbalanced methylation and changes of allelic expression induced by hyperhomocysteinaemia in patients with uraemia. Lancet, 361, 1693-1699.

31. Sakatani, T., Kaneda, A., Iacobuzio-Donahue, C.A., Carter, M.G., de Boom Witzel, S., Okano, H., Ko, M.S., Ohlsson, R., Longo, D.L. and Feinberg, A.P. (2005) Loss of imprinting of Igf2 alters intestinal maturation and tumorigenesis in mice. Science, 307, 1976-1978. 
32. Wang, W.H., Duan, J.X., Vu, T.H. and Hoffman, A.R. (1996) Increased expression of the insulin-like growth factor-II gene in Wilms' tumor is not dependent on loss of genomic imprinting or loss of heterozygosity. J. Biol. Chem., 271, 27863-27870.

33. Rainier, S., Johnson, L.A., Dobry, C.J., Ping, A.J., Grundy, P.E. and Feinberg, A.P. (1993) Relaxation of imprinted genes in human cancer. Nature, 362, 747-749.

34. Forne, T., Oswald, J., Dean, W., Saam, J.R., Bailleul, B., Dandolo, L., Tilghman, S.M., Walter, J. and Reik, W. (1997) Loss of the maternal H19 gene induces changes in Igf2 methylation in both cis and trans. Proc. Natl Acad. Sci. USA, 94, 10243-10248.

35. Briere, N. (1988) Effect of hormones on hydrolase activities and DNA synthesis in kidney of the developing mouse. Can. J. Physiol. Pharmacol., 66, 580-585.

36. Pogribny, I.P. and James, S.J. (2002) De novo methylation of the p16INK4A gene in early preneoplastic liver and tumors induced by folate/ methyl deficiency in rats. Cancer Lett., 187, 69-75.

37. Tremolizzo, L., Carboni, G., Ruzicka, W.B., Mitchell, C.P., Sugaya, I., Tueting, P., Sharma, R., Grayson, D.R., Costa, E. and Guidotti, A. (2002) An epigenetic mouse model for molecular and behavioral neuropathologies related to schizophrenia vulnerability. Proc. Natl Acad. Sci. USA, 99, 17095-170100.

38. Lucas, A. (1998) Programming by early nutrition: an experimental approach. J. Nutr., 128, 401S-406S.

39. Jirtle, R.L. (2004) IGF2 loss of imprinting: a potential heritable risk factor for colorectal cancer. Gastroenterology, 126, 1190-1193.
40. Bateson, P., Barker, D., Clutton-Brock, T., Deb, D., D’Udine, B., Foley, R.A., Gluckman, P., Godfrey, K., Kirkwood, T., Lahr, M.M. et al. (2004) Developmental plasticity and human health. Nature, 430, 419-421.

41. Wainfan, E., Dizik, M., Stender, M. and Christman, J.K. (1989) Rapid appearance of hypomethylated DNA in livers of rats fed cancerpromoting, methyl-deficient diets. Cancer Res., 49, 4094-4097.

42. Strauss, W.M. (2001) Preparation of genomic DNA from mammalian tissue. In Ausubel, F.M. et al. (eds), Current Protocols in Molecular Biology. Vol. 1. Wiley, New York, pp. 2.2.1-2.2.3.

43. Moore, T., Constancia, M., Zubair, M., Bailleul, B., Feil, R., Sasaki, H. and Reik, W. (1997) Multiple imprinted sense and antisense transcripts, differential methylation and tandem repeats in a putative imprinting control region upstream of mouse Igf2. Proc. Natl Acad. Sci. USA, 94, 12509-12514.

44. Eden, S., Constancia, M., Hashimshony, T., Dean, W., Goldstein, B., Johnson, A.C., Keshet, I., Reik, W. and Cedar, H. (2001) An upstream repressor element plays a role in Igf2 imprinting. EMBOJ., 20, 3518-3525.

45. Pettersson, M., Bylund, M. and Alderborn, A. (2003) Molecular haplotype determination using allele-specific PCR and pyrosequencing technology. Genomics, 82, 390-396.

46. Murrell, A., Heeson, S., Bowden, L., Constancia, M., Dean, W., Kelsey, G. and Reik, W. (2001) An intragenic methylated region in the imprinted Igf2 gene augments transcription. EMBO Rep., 2, 1101-1106.

47. Herbig, K., Chiang, E.P., Lee, L.R., Hills, J., Shane, B. and Stover, P.J. (2002) Cytoplasmic serine hydroxymethyltransferase mediates competition between folate-dependent deoxyribonucleotide and S-adenosylmethionine biosyntheses. J. Biol. Chem., 277, 38381-38389. 